\title{
Reflection coefficient of a dominant mode in a pentafurcated duct
}

\author{
Mahmood-ul-Hassan and Mahvish Naz
}

"Correspondence:

mahvish.naz1@gmail.com

Mathematics Department,

COMSATS Institute of Information

Technology, Park Road, Chak

Shahzad, Islamabad, Pakistan

\begin{abstract}
This paper presents the diffraction of acoustic plane wave through a semi-infinite soft duct. The soft plates are enclosed symmetrically inside an infinite duct with hard boundary conditions. We fully emphasize the analysis of the behavior of the reflected field amplitude for the pentafurcated duct by applying the straightforward mode-matching technique. We also depict some graphical representations by determining the reflected field amplitude for various dimensions of the pentafurcated duct. We also present comparisons of results with the existing results of a hard pentafurcated duct problem. This research work will assist the future researchers in reducing noise effects in complicated devices and exhaust systems.
\end{abstract}

Keywords: scattering; acoustic; reflected field; pentafurcated waveguide; energy balance

\section{Introduction}

In the 20th century, excessive amount of noise and unpleasant sounds became a part of the urban culture that caused temporary disturbance in the natural balance. In urban areas, many industries use big machines and devices which produce excessive sounds and contribute to noise pollution. In addition to this, different equipments and vehicles, like compressors, generators, exhaust fans, grinding mills etc., contribute to the production of unwanted noises. In the past thirty years, unwanted noises have become a major threat to physical and mental health of people, especially for those residing in urban areas. In many technical and industrial fields, a variety of mechanisms have become the source of harmful and undesired noise. Structural acoustics have provided a large number of challenging and interesting problems for applied mathematicians, physicists and engineers. That is the reason why it has become a topic of great interest to study the diffraction of sound wave in various structures with different combinations of the boundary conditions (soft, hard, impedance etc.).

Over the years, many researchers have analyzed different types of structures involving different boundary conditions by integral transform and Jones method based on the Wiener-Hopf technique.

Buyukaksoy et al. [1] studied a bifurcated cylindrical waveguide with wall impedance discontinuity. Rawlins [2] also analyzed the solution of a bifurcated circular waveguide problem. Mahmood-ul-Hassan and Rawlins [3] analyzed two problems of waveguides carrying mean flow. They also defined a planar trifurcated lined duct having sound radiations

(c) The Author(s) 2017. This article is distributed under the terms of the Creative Commons Attribution 4.0 International License (http://creativecommons.org/licenses/by/4.0/), which permits unrestricted use, distribution, and reproduction in any medium, provided you give appropriate credit to the original author(s) and the source, provide a link to the Creative Commons license, and indicate if changes were made. 
[4]. They solved all these problems by applying the Wiener-Hopf technique. Ayub et al. [5-7] presented acoustic diffraction in a trifurcated waveguide with different boundary conditions (hard, soft, impedance etc.). Demir and Cinar [8] considered the propagation of sound in a two-part circular cylindrical duct carrying a mean gas flow, inserted in a larger infinite outer duct with wall impedance discontinuity. All researchers mentioned above solved these problems by applying the Wiener-Hopf technique. The solution obtained by the Wiener-Hopf technique consists of complicated factors or split functions. Sometimes, it is very complicated to handle the matrix Wiener-Hopf problem.

Keeping in mind past research work, we emphasize the mode-matching technique which is powerful, straightforward and simple compared to Wiener-Hopf and other techniques. In this technique, potential solutions which satisfy the appropriate boundary conditions are matched across the boundary of the structure. Many researchers have used the mode-matching technique to solve various structures with different boundary conditions. Andronor and Belinskii [9] proposed the diffraction field of acoustic waves in a vertically stratified medium covered from above by an elastic plate by using the integral transformation technique. Lawrie et al. [10-12] analyzed different types of geometries by applying the mode-matching technique to get solutions by developing new orthogonality relations. Lawrie [13] solved geometry involving scattering in three-dimensional waveguide with flexible wall by using the mode-matching technique. Mahmood-ul-Hassan et al. [14] analyzed water wave scattering in a submerged elastic plate by applying the modematching technique. Nawaz and Lawrie [15] investigated the scattering of a wave at a flanged junction between two flexible waveguides by using the mode-matching technique. The flanged junction has one side soft and another rigid. Decrossas et al. [16] analyzed the modeling of coaxial circular waveguide discontinuities for material characterization purposes by applying the mode-matching technique. Ranajit et al. [17] presented the design and analysis of cubic spline interpolated profiled smooth-wall multi-mode horn using the mode-matching technique along with evolutionary algorithm. Meylan et al. [18] developed an eigenfunction expansion method for the problem of linear water-wave scattering by a circular floating porous elastic plate and also developed a coupled boundary-element and finite element plate shape. Sanchis et al. [19] reported a mode-matching technique for highly efficient coupling between dielectric silica waveguides and planar photonic crystal waveguides based on setting localized defects in a PPC tapered waveguide. Hassan [20] analyzed the propagation of the lowest mode from semi-infinite soft-hard three spaced duct by applying the straightforward mode-matching technique. Hassan et al. [21] solved a complicated hard pentafurcated duct problem by applying the powerful mode-matching technique. The related work on a soft-hard pentafurcated waveguide problem has been tackled by Hassan et al. [22] using the straightforward mode-matching technique.

We consider here a pentafurcated problem with outer hard and inner soft boundary conditions. The soft linings (the pressure release conditions) are introduced to see the effect on the reflected field with a hard pentafurcated problem [21]. The given problem provides a good first hand approximation in the actual system. This prototype model will be helpful for researchers to deal with more general conditions. We split our pentafurcated structure into six regions. The potential solution is obtained in an individual region by applying the separation of variables method. The orthogonality relations permit the given problem to be reduced in the form of infinite systems of linear algebraic equations. These infinite systems of equations are solved by applying MATLAB programming. The 
Richardson extrapolation formula is used to estimate the errors in the solution. The linear systems of equations converge, so we can truncate the system of equations in our calculation. We depict the reflected field amplitude graphically for various dimensions of the given waveguide. We also present the comparison of the acoustic energy which is proportional to $\left(1-|R|^{2}\right)$ for the current problem with an existing hard pentafurcated duct which was studied by Hassan et al. [21]. This article will be helpful for engineers in the future to construct exhaust systems to attenuate noise pollution.

We arrange this article in the following manner. In Section 2, the model problem is formulated, while in Section 3 mode-matching solutions are presented. The convergence of the given problem is synchronized in Section 4. In Section 5, some numerical results are explained and represented graphically. The comparison between the current problem and the hard pentafurcated problem [21] is shown in Section 6. In Section 7, the energy conservation is also determined to derive power balance relationship between different coefficients of reflection and transmission. The final remarks are given in Section 8.

\section{Formulation of the boundary value problem}

We consider the diffraction of an incident plane mode from a semi-infinite soft surface duct $(|y|<a, x<0)$ which has geometrical representation in Figure 1. We form a twodimensional pentafurcated structure such that four semi-infinite soft plates are located inside two infinite hard plates. Infinite hard plates are located at $y= \pm d$.

We introduce the scalar potential function $\varphi(x, y, t)$ and define acoustic pressure by $p=$ $-\rho_{o} \frac{\partial \varphi}{\partial t}$ and velocity vector by $\vec{u}=\operatorname{grad}(\varphi)$, respectively (where $\rho_{o}$ indicates the density in equilibrium state). The potential $\varphi$ satisfies the following wave equation:

$$
\nabla^{2} \varphi=\left(1 / c^{2}\right) \varphi_{t t} .
$$

We assume

$$
\varphi(x, y, t)=\operatorname{Re}\left[v(x, y) e^{-i w t}\right]
$$

where $c$ is the speed of the sound, $w$ is angular frequency, and the wave number is defined as $k=\frac{w}{c}$. Equation (2.2) identically satisfies equation (2.1) which eventually results into a well-known Helmholtz equation in $2 D$ that is within the duct

$$
\left(\nabla^{2}+k^{2}\right) v(x, y)=0
$$

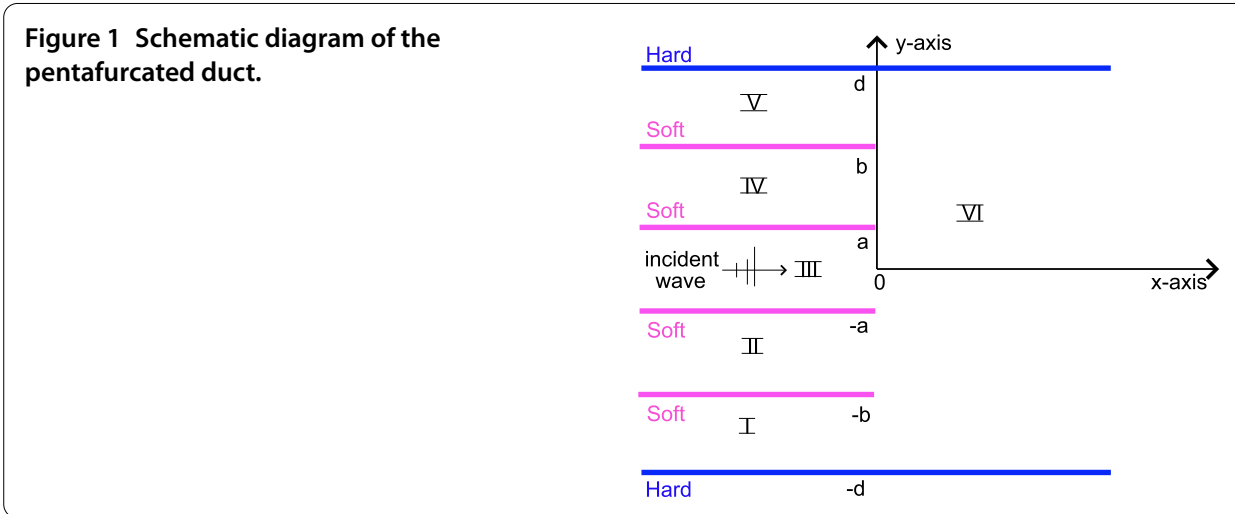


We will solve the problem subject to the following boundary conditions:

$$
\begin{aligned}
& v_{y}(x, y)=0, \quad y= \pm d,-\infty<x<\infty \\
& v(x, y)=0, \quad y= \pm b,-\infty<x<0 \\
& v(x, y)=0, \quad y= \pm a,-\infty<x<0 .
\end{aligned}
$$

The wave field satisfies the radiation conditions:

$$
v(x, y)-v^{i}(x, y) \text { is outgoing and bounded as } x \rightarrow \pm \infty,
$$

which simply ensures the boundedness of the obtained solution. In order to ensure the unique solution, different extra conditions, which are termed as edge conditions, are imposed. However, the problem is well posed as mentioned above. Therefore, no extra condition is required for the uniqueness of the solutions. Yet, in the case of higher order boundaries, the use of edge conditions becomes relevant. This statement is well supported through a number of research articles (see, for instance, [15, 23]).

\subsection{Region I: $\{-d \leq y \leq-b, x<0\}$}

In the given problem, the potential solution is obtained in each region by applying the method of separation of variables. Then we use the straightforward eigenfunction expansion technique proposed by Mei [24] and Linton \& Mclver [25].

The potential solution of equation (2.3) in region I can be written as

$$
v(x, y)=\sum_{n=1}^{\infty} A_{n} e^{-i \hat{\alpha}_{n} x}\left(\sqrt{\frac{2}{(d-b)}} \sin \alpha_{n}(y+b)\right),
$$

which satisfies equations (2.4), (2.5) and radiation conditions (2.7), where $A_{n}$ represents transmitted field amplitudes in region I.

The orthonormal relation is defined as

$$
\begin{aligned}
& \int_{-d}^{-b}\left(\sqrt{\frac{2}{(d-b)}} \sin \alpha_{m}(y+b)\right)\left(\sqrt{\frac{2}{(d-b)}} \sin \alpha_{n}(y+b)\right) d y \\
& \quad=\delta_{m n}, \quad m, n=1,2,3, \ldots,
\end{aligned}
$$

where $\delta_{m n}$ is the Kronecker delta defined by

$$
\delta_{m n}= \begin{cases}0, & m \neq n, \\ 1, & m=n .\end{cases}
$$

The associated eigenvalues are

$$
\begin{aligned}
& \hat{\alpha}_{1}=\sqrt{k^{2}-\left(\frac{\pi}{2(d-b)}\right)^{2}}, \\
& \hat{\alpha}_{2}=\sqrt{k^{2}-\left(\frac{3 \pi}{2(d-b)}\right)^{2}}
\end{aligned}
$$




$$
\hat{\alpha}_{n}=\sqrt{k^{2}-\left(\frac{(2 n-1) \pi}{2(d-b)}\right)^{2}}, \quad n=1,2,3, \ldots
$$

with $0<\operatorname{Im} \hat{\alpha}_{1}<\operatorname{Im} \hat{\alpha}_{2} \ldots$ and $\operatorname{Re} \hat{\alpha}_{n}>0$.

The eigenvalues $\alpha_{n}=\frac{(2 n-1) \pi}{2(d-b)}$ are the solution of the following relation:

$$
\cos \alpha_{n}(d-b)=0, \quad n=1,2,3, \ldots
$$

\subsection{Region II: $\{-b \leq y \leq-a, x<0\}$}

The potential solution of equation (2.3) in this region is given by

$$
v(x, y)=\sum_{n=1}^{\infty} B_{n} e^{-i \hat{\beta}_{n} x}\left(\sqrt{\frac{2}{(b-a)}} \sin \beta_{n}(y+b)\right),
$$

which satisfies equations (2.5), (2.6) and radiation conditions (2.7), where $B_{n}$ is the amplitudes of transmitted field in region II.

The eigenfunctions satisfy the orthonormal relation

$$
\begin{aligned}
& \int_{-b}^{-a}\left(\sqrt{\frac{2}{(b-a)}} \sin \beta_{m}(y+b)\right)\left(\sqrt{\frac{2}{(b-a)}} \sin \beta_{n}(y+b)\right) d y \\
& \quad=\delta_{m n}, \quad m, n=1,2,3, \ldots
\end{aligned}
$$

The associated eigenvalues are

$$
\begin{aligned}
& \hat{\beta}_{1}=\sqrt{k^{2}-\left(\frac{\pi}{(b-a)}\right)^{2}}, \\
& \hat{\beta}_{2}=\sqrt{k^{2}-\left(\frac{2 \pi}{(b-a)}\right)^{2}} \\
& \vdots \\
& \hat{\beta}_{n}=\sqrt{k^{2}-\left(\frac{n \pi}{(b-a)}\right)^{2}}, \quad n=1,2,3, \ldots,
\end{aligned}
$$

with $0<\operatorname{Im} \hat{\beta}_{1}<\operatorname{Im} \hat{\beta}_{2} \ldots$ and $\operatorname{Re} \hat{\beta}_{n}>0$.

The eigenvalues $\beta_{n}=\frac{n \pi}{(b-a)}$ satisfy the equation

$$
\sin \beta_{n}(b-a)=0, \quad n=1,2,3, \ldots
$$

\subsection{Region III: $\{-a \leq y \leq a, x<0\}$}

The potential solution of equation (2.3) in region III is defined as

$$
v(x, y)=\sum_{n=1}^{\infty} C_{n} e^{-i \hat{\gamma}_{n} x}\left(\sqrt{\frac{1}{a}} \sin \gamma_{n}(y+a)\right)+e^{\hat{\gamma}_{1} x}\left(\sqrt{\frac{1}{a}} \sin \gamma_{1}(y+a)\right),
$$


which also satisfies equation (2.6) and radiation conditions (2.7), where the incident wave $e^{i \hat{\gamma}_{1} x}\left(\sqrt{\frac{1}{a}} \sin \gamma_{1}(y+a)\right)$ is excited in the lowest mode propagating from $x=-\infty$.

The eigenfunctions in this region define the orthonormal relation

$$
\int_{-a}^{a}\left(\sqrt{\frac{1}{a}} \sin \gamma_{m}(y+a)\right)\left(\sqrt{\frac{1}{a}} \sin \gamma_{n}(y+a)\right) d y=\delta_{m n}, \quad m, n=1,2,3, \ldots
$$

The associated eigenvalues are

$$
\begin{aligned}
& \hat{\gamma}_{1}=\sqrt{k^{2}-\left(\frac{\pi}{2 a}\right)^{2}}, \\
& \hat{\gamma}_{2}=\sqrt{k^{2}-\left(\frac{\pi}{a}\right)^{2}} \\
& \vdots \\
& \hat{\gamma}_{n}=\sqrt{k^{2}-\left(\frac{n \pi}{2 a}\right)^{2}}, \quad n=1,2,3, \ldots
\end{aligned}
$$

with $0<\operatorname{Im} \hat{\gamma}_{1}<\operatorname{Im} \hat{\gamma}_{2} \ldots$ and $\operatorname{Re} \hat{\gamma}_{n}>0$.

The eigenvalues $\gamma_{n}=\frac{n \pi}{2 a}$ are the roots of the equation

$$
\sin \left(\gamma_{n} 2 a\right)=0, \quad n=1,2,3, \ldots
$$

\subsection{Region IV: $\{a \leq y \leq b, x<0\}$}

The general potential solution of equation (2.3) in region IV is defined as

$$
v(x, y)=\sum_{n=1}^{\infty} D_{n} e^{-i \hat{\beta}_{n} x}\left(\sqrt{\frac{2}{(b-a)}} \sin \beta_{n}(y-a)\right),
$$

which satisfies equations (2.5), (2.6) and radiation conditions (2.7), where $D_{n}$ is the amplitudes of transmitted field in region IV.

The eigenfunctions satisfy the orthonormal relation

$$
\begin{aligned}
& \int_{a}^{b}\left(\sqrt{\frac{2}{(b-a)}} \sin \beta_{m}(y-a)\right)\left(\sqrt{\frac{2}{(b-a)}} \sin \beta_{n}(y-a)\right) d y \\
& \quad=\delta_{m n}, \quad m, n=1,2,3, \ldots
\end{aligned}
$$

\subsection{Region V: $\{b \leq y \leq d, x<0\}$}

The general potential solution of equation (2.3) in this region is defined as

$$
v(x, y)=\sum_{n=1}^{\infty} E_{n} e^{-i \hat{\alpha}_{n} x}\left(\sqrt{\frac{2}{(d-b)}} \sin \alpha_{n}(y-b)\right),
$$

which satisfies equations (2.4), (2.5) and radiation conditions (2.7), where $E_{n}$ is the amplitudes of transmitted field in region $\mathrm{V}$. 
The eigenfunctions satisfy the orthonormal relation

$$
\begin{aligned}
& \int_{b}^{d}\left(\sqrt{\frac{2}{(d-b)}} \sin \alpha_{m}(y-b)\right)\left(\sqrt{\frac{2}{(d-b)}} \sin \alpha_{n}(y-b)\right) d y \\
& \quad=\delta_{m n}, \quad m, n=1,2,3, \ldots
\end{aligned}
$$

\subsection{Region VI: $\{-d \leq y \leq d, x>0\}$}

The general potential solution of equation (2.3) in region VI is defined as

$$
v(x, y)=\sum_{n=1}^{\infty} F_{n} e^{i \hat{\lambda}_{n} x} \bar{\Psi}_{n}^{(\mathrm{VI})}(y) d y
$$

which satisfies equation (2.4) and radiation conditions (2.7).

We define the vertical orthonormal eigenfunctions in region VI as

$$
\bar{\Psi}_{n}^{(\mathrm{VI})}(y) d y= \begin{cases}\sqrt{\frac{1}{2 d}}, & \text { if } n=1, \\ \sqrt{\frac{1}{d}} \cos \lambda_{n}(y+d), & \text { if } n \neq 1 .\end{cases}
$$

The associated eigenvalues are

$$
\begin{aligned}
& \hat{\lambda}_{2}=\sqrt{k^{2}-\left(\frac{\pi}{2 d}\right)^{2}}, \\
& \hat{\lambda}_{3}=\sqrt{k^{2}-\left(\frac{\pi}{d}\right)^{2}} \\
& \vdots \\
& \hat{\lambda}_{n}=\sqrt{k^{2}-\left(\frac{(n-1) \pi}{2 d}\right)^{2}}, \quad n=1,2,3, \ldots,
\end{aligned}
$$

with $0<\operatorname{Im} \hat{\lambda}_{1}<\operatorname{Im} \hat{\lambda}_{2} \ldots$ and $\operatorname{Re} \hat{\lambda}_{n}>0$.

The eigenvalues $\lambda_{n}=\frac{(n-1) \pi}{2 d}$ satisfy the equation

$$
\sin \left(\lambda_{n} 2 d\right)=0, \quad n=1,2,3, \ldots
$$

\section{Solution of the pentafurcated waveguide problem}

Here, we formulate an infinite system of equations by using potential solutions in all regions which are matched at $x=0$ to exploit the continuity of potentials and the continuity of derivative of potentials (see Figure 1). Then we solve these system of equations by numerically truncating them.

\subsection{Continuity of pressure}

The continuity of pressure in region I and region VI at $x=0$ gives

$$
\sum_{n=1}^{\infty} A_{n}\left(\sqrt{\frac{2}{(d-b)}} \sin \alpha_{n}(y+b)\right)=\sum_{n=1}^{\infty} F_{n} \bar{\Psi}_{n}^{(\mathrm{VI})}(y) .
$$


On taking the inner product with $\left(\sqrt{\frac{2}{(d-b)}} \sin \alpha_{m}(y+b)\right)$, integrating over $[-d,-b]$ and using equation (2.9), we obtain

$$
A_{m}=\sum_{n=1}^{\infty} F_{n} P_{m n}, \quad m=1,2,3, \ldots
$$

where

$$
\begin{aligned}
P_{m n} & =\int_{-d}^{-b}\left(\sqrt{\frac{2}{(d-b)}} \sin \alpha_{m}(y+b)\right) \bar{\Psi}_{n}^{(\mathrm{VI})}(y) d y \\
& = \begin{cases}\sqrt{\frac{2}{d(d-b)}}\left[\frac{-\alpha_{m} \cos \lambda_{n}(d-b)}{\alpha_{m}^{2}-\lambda_{n}^{2}}\right], & \text { if } \lambda_{n} \neq \alpha_{m}, \\
\sqrt{\frac{1}{2 d(d-b)}}\left[(d-b) \sin \alpha_{m}(b-d)\right], & \text { if } \lambda_{n}=\alpha_{m}, \\
-\frac{1}{\alpha_{m} \sqrt{d(d-b)}}, & \text { if } n=1, m \neq 1 .\end{cases}
\end{aligned}
$$

The continuity of pressure in region II and region VI across $x=0$ gives

$$
\sum_{n=1}^{\infty} B_{n}\left(\sqrt{\frac{2}{(b-a)}} \sin \beta_{n}(y+b)\right)=\sum_{n=1}^{\infty} F_{n} \bar{\Psi}_{n}^{(\mathrm{VI})}(y) .
$$

Again by taking the inner product with $\left(\sqrt{\frac{2}{(b-a)}} \sin \beta_{m}(y+b)\right)$, integrating over $[-b,-a]$ and using equation (2.17), we obtain

$$
B_{m}=\sum_{n=1}^{\infty} F_{n} Q_{m n}, \quad m=1,2,3, \ldots
$$

where

$$
\begin{aligned}
Q_{m n} & =\int_{-b}^{-a}\left(\sqrt{\frac{2}{(b-a)}} \sin \beta_{m}(y+b)\right) \bar{\Psi}_{n}^{(\mathrm{VI})}(y) d y \\
& = \begin{cases}\sqrt{\frac{2}{d(b-a)}}\left[\frac{\beta_{m}\left(\cos \lambda_{n}(d-b)-\cos \beta_{m}(b-a) \cos \lambda_{n}(d-a)\right)}{\beta_{m}^{2}-\lambda_{n}^{2}}\right], & \text { if } \lambda_{n} \neq \beta_{m}, \\
\sqrt{\frac{1}{2 d(b-a)}}\left[\frac{\cos \beta_{m}(d-b)-\cos \beta_{m}(b+d-2 a)}{2 \beta_{m}}+(b-a) \sin \beta_{m}(b-d)\right], & \text { if } \lambda_{n}=\beta_{m}, \\
\sqrt{\frac{1}{d(b-a)}}\left[\frac{1-\cos \beta_{m}(b-a)}{\beta_{m}}\right], & \text { if } n=1, m \neq 1 .\end{cases}
\end{aligned}
$$

The continuity of pressure in region III and region VI at $x=0$ gives

$$
\sum_{n=1}^{\infty} C_{n}\left(\sqrt{\frac{1}{a}} \sin \gamma_{n}(y+a)\right)+\left(\sqrt{\frac{1}{a}} \sin \gamma_{1}(y+a)\right)=\sum_{n=1}^{\infty} F_{n} \bar{\Psi}_{n}^{(\mathrm{VI})}(y) .
$$

On taking the inner product with $\left(\sqrt{\frac{1}{a}} \sin \gamma_{m}(y+a)\right)$, integrating over $[-a, a]$ and using (2.24), we get

$$
C_{m}+\delta_{1 m}=\sum_{n=1}^{\infty} F_{n} R_{m n}, \quad m=1,2,3, \ldots
$$


where

$$
\begin{aligned}
R_{m n} & =\int_{-a}^{a}\left(\sqrt{\frac{1}{a}} \sin \gamma_{m}(y+a)\right) \bar{\Psi}_{n}^{(\mathrm{VI})}(y) d y \\
& = \begin{cases}\sqrt{\frac{1}{a d}}\left[\frac{\gamma_{m}\left(\cos \lambda_{n}(d-a)-\cos \gamma_{m}^{2} 2 a \cos \lambda_{n}(a+d)\right)}{\gamma_{m}^{2}-\lambda_{n}^{2}}\right], & \text { if } \lambda_{n} \neq \gamma_{m}, \\
\frac{1}{2 \sqrt{a d}}\left[\frac{\cos \gamma_{m}(d-a)-\cos \gamma_{m}(3 a+d)}{2 \gamma_{m}}+2 a \sin \gamma_{m}(a-d)\right], & \text { if } \lambda_{n}=\gamma_{m}, \\
\frac{1}{\sqrt{2 a d}}\left[\frac{1-\cos \gamma_{m} 2 a}{\gamma_{m}}\right], & \text { if } n=1, m \neq 1 .\end{cases}
\end{aligned}
$$

The continuity of pressure in region IV and region VI across $x=0$ gives

$$
\sum_{n=1}^{\infty} D_{n}\left(\sqrt{\frac{2}{(b-a)}} \sin \beta_{n}(y-a)\right)=\sum_{n=1}^{\infty} F_{n} \bar{\Psi}_{n}^{(\mathrm{VI})}(y) .
$$

While taking the inner product with $\left(\sqrt{\frac{2}{(b-a)}} \sin \beta_{m}(y-a)\right)$, integrating over $[a, b]$ and using (2.31), we obtain

$$
D_{m}=\sum_{n=1}^{\infty} F_{n} S_{m n}, \quad m=1,2,3, \ldots
$$

where

$$
\begin{aligned}
S_{m n} & =\int_{a}^{b}\left(\sqrt{\frac{2}{(b-a)}} \sin \beta_{m}(y-a)\right) \bar{\Psi}_{n}^{(\mathrm{VI})}(y) d y \\
& = \begin{cases}\sqrt{\frac{2}{d(b-a)}}\left[\frac{\beta_{m} \cos \lambda_{n}(a+d)-\beta_{m} \cos \lambda_{n}(b+d) \cos \beta_{m}(b-a)}{\beta_{m}^{2}-\lambda_{n}^{2}}\right], & \text { if } \lambda_{n} \neq \beta_{m}, \\
\frac{1}{\sqrt{2 d(b-a)}}\left[\frac{\cos \beta_{m}(a+d)-\cos \beta_{m}(2 b+d-a)}{2 \beta_{m}}-(b-a) \sin \beta_{m}(a+d)\right], & \text { if } \lambda_{n}=\beta_{m}, \\
\frac{1}{\sqrt{d(b-a)}}\left[\frac{1-\cos \beta_{m}(b-a)}{\beta_{m}}\right], & \text { if } n=1, m \neq 1 .\end{cases}
\end{aligned}
$$

The continuity of pressure in region $\mathrm{V}$ and region $\mathrm{VI}$ across $x=0$ gives

$$
\sum_{n=1}^{\infty} E_{n}\left(\sqrt{\frac{2}{(d-b)}} \sin \alpha_{n}(y-b)\right)=\sum_{n=1}^{\infty} F_{n} \bar{\Psi}_{n}^{(\mathrm{VI})}(y) .
$$

Taking the inner product with $\left(\sqrt{\frac{2}{(d-b)}} \sin \alpha_{m}(y-b)\right)$, integrating over $[b, d]$ and using (2.33), we obtain

$$
E_{m}=\sum_{n=1}^{\infty} F_{n} T_{m n}, \quad m=1,2,3, \ldots,
$$

where

$$
\begin{aligned}
T_{m n} & =\int_{b}^{d}\left(\sqrt{\frac{2}{(d-b)}} \sin \alpha_{m}(y-b)\right) \bar{\Psi}_{n}^{(\mathrm{VI})} d y \\
& = \begin{cases}\sqrt{\frac{2}{d(d-b)}}\left[\frac{\alpha_{m} \cos \lambda_{n}(b+d)-\lambda_{n} \sin \lambda_{n} 2 d \sin \alpha_{m}(d-b)}{\alpha_{m}^{2}-\lambda_{n}^{2}}\right], & \text { if } \lambda_{n} \neq \alpha_{m}, \\
\frac{1}{\sqrt{2 d(d-b)}}\left[\frac{\cos \alpha_{m}(b+d)-\cos \alpha_{m}(3 d-b)}{2 \alpha_{m}}-(d-b) \sin \alpha_{m}(b+d)\right], & \text { if } \lambda_{n}=\alpha_{m}, \\
\frac{1}{\alpha_{m} \sqrt{d(d-b)}}, & \text { if } n=1, m \neq 1 .\end{cases}
\end{aligned}
$$




\subsection{Continuity of velocity}

Whilst following the working procedure of subsection (3.1), the continuity of the derivatives of the potential solutions in different regions across $x=0$ give the following systems of equations.

$$
\begin{aligned}
& -\hat{\alpha}_{m} A_{m}=\sum_{n=1}^{\infty} \hat{\lambda}_{n} F_{n} P_{m n}, \quad m=1,2,3, \ldots, \\
& -\hat{\beta}_{m} B_{m}=\sum_{n=1}^{\infty} \hat{\lambda}_{n} F_{n} Q_{m n}, \quad m=1,2,3, \ldots, \\
& \hat{\gamma}_{1} \delta_{1 m}-\hat{\gamma}_{m} C_{m}=\sum_{n=1}^{\infty} \hat{\lambda}_{n} F_{n} R_{m n}, \quad m=1,2,3, \ldots, \\
& -\hat{\beta}_{m} D_{m}=\sum_{n=1}^{\infty} \hat{\lambda}_{n} F_{n} S_{m n}, \quad m=1,2,3, \ldots, \\
& -\hat{\alpha}_{m} E_{m}=\sum_{n=1}^{\infty} \hat{\lambda}_{n} F_{n} T_{m n}, \quad m=1,2,3, \ldots
\end{aligned}
$$

\subsection{Coupling}

Although we can solve equations (3.1) to (3.10) for unknown coefficients, we prefer to couple these systems of equations for the sake of convenience. From (3.1) and (3.6), we have

$$
\sum_{n=1}^{\infty} F_{n} P_{m n}\left(\hat{\alpha}_{m}+\hat{\lambda}_{n}\right)=0, \quad m=1,2,3, \ldots
$$

where equations (3.2) and (3.7) yield

$$
\sum_{n=1}^{\infty} F_{n} Q_{m n}\left(\hat{\beta}_{m}+\hat{\lambda}_{n}\right)=0, \quad m=1,2,3, \ldots
$$

From (3.3) and (3.8), we have

$$
\sum_{n=1}^{\infty} F_{n} R_{m n}\left(\hat{\gamma}_{m}+\hat{\lambda}_{n}\right)=\left(\hat{\gamma}_{1}+\hat{\gamma}_{m}\right) \delta_{1 m}, \quad m=1,2,3, \ldots
$$

equations (3.4) and (3.9) give

$$
\sum_{n=1}^{\infty} F_{n} S_{m n}\left(\hat{\beta}_{m}+\hat{\lambda}_{n}\right)=0, \quad m=1,2,3, \ldots
$$

From (3.5) and (3.10), we have

$$
\sum_{n=1}^{\infty} F_{n} T_{m n}\left(\hat{\alpha}_{m}+\hat{\lambda}_{n}\right)=0, \quad m=1,2,3, \ldots
$$



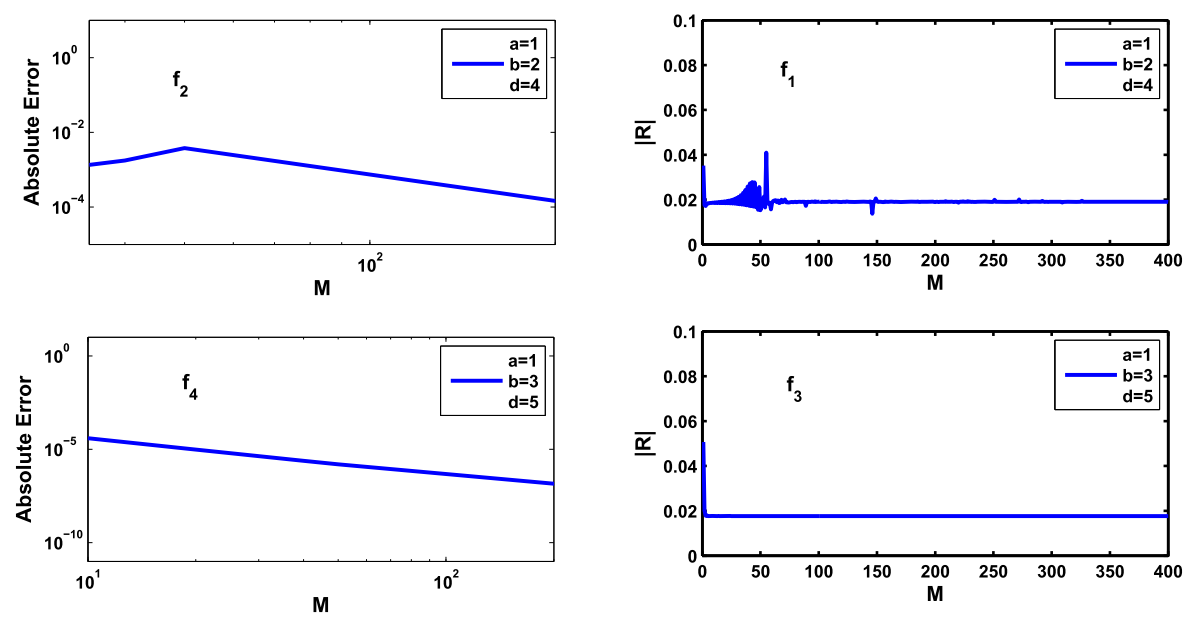

Figure 2 Reflected field amplitude $|R|$ and absolute error versus the truncation number $M$.

\section{Convergence}

Now we simplify an infinite system of equations to obtain unknown coefficients from region I to region VI. We plot the reflected field amplitude $|R|$ and the absolute error of reflection coefficient by exploiting the Richardson extrapolation formula as a function of truncation number $M$ for different dimensions of the duct having frequency $\pi$ as shown in Figure 2. We can observe clearly that the reflection becomes insensitive when $M \geq 70$ for both dimensions of the duct as presented in Figure 2 of sub-graphs $f_{1}$ and $f_{3}$. In order to estimate the exact solution, the Richardson extrapolation is used for large values of $\mathrm{M}$ $(M=400$ and $N=5 M)$ as represented in the sub-graphs $f_{2}$ and $f_{4}$ of Figure 2. The infinite system of equations converges so that we can truncate at $M \approx 100$, which indicates the errors within the line width.

\section{Numerical results}

Now, we truncate the infinite system of equations (3.11)-(3.15) by taking $n=1,2,3, \ldots, 5 \mathrm{M}$ and $m=1,2,3, \ldots, M$ to solve $F_{n}$. To check out the intensity of transmitted wave for the current problem, we consider the reflection coefficient since the transmitted acoustic energy is proportional to $\left(1-|R|^{2}\right)$. In Figures 3-6, we present the graphical representation of the reflected field $|R|=\left|C_{1}\right|$ versus the wave number $k$ for different dimensions of the duct. We assume only one propagating wave in each region $I-V$, then we observe the propagating modes in region VI.

In Figure 3, we consider that the dimensions of coaxial regions $(b<|y|<d, a<|y|<b$, $x<0)$ are such that $b=3, d=5$, while the width of the middle region $(|y|<a, x<0)$ has values $a=1$ and $a=2$. The frequency range is $0<k<\pi$. Three possible modes can propagate in the forward direction in region $\mathrm{VI}(|y|<d, x>0)$ from $(\pi / 2<k<3 \pi / 5,3 \pi / 5<k<4 \pi / 5$, $4 \pi / 5<k<\pi)$ for $a=1$. There are two modes in the forward direction for $a=2$ in this condition. We can see from the graphical representation clearly that the cut-off(on) frequency is changed from $\pi / 4$ to $\pi / 2$ as the width of the middle region is decreased. We also observe that the absolute value of reflection coefficient increases as the dimension of the inner most duct decreases, while the width of the other coaxial regions is fixed. 


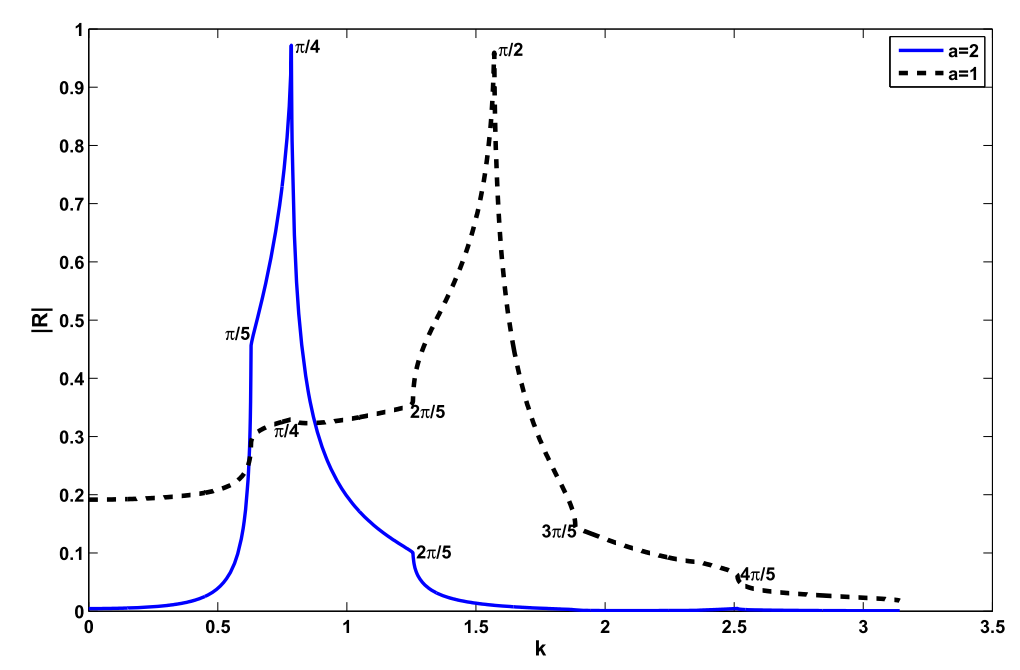

Figure 3 Reflected field amplitude $|R|$ versus the wave number $k$ for $b=3$ and $d=5$.

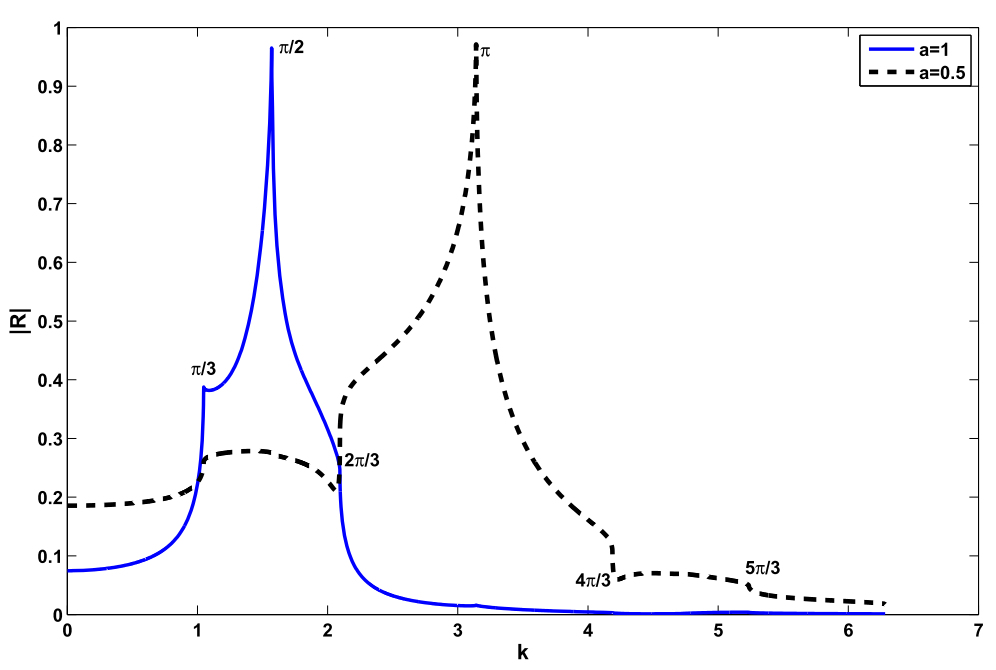

Figure 4 Reflected field amplitude $|R|$ versus the wave number $k$ for $b=2$ and $d=3$.

In Figure 4, we assume small values $a=0.5$ and $a=1$ for the width of the middle region $(x<0,|y|<a)$. The frequency range is $0<k<2 \pi$. The values of coaxial regions are kept fixed $b=2$ and $d=3$. The cut-on frequency is changed from $\pi / 2$ to $\pi$ as the width of the inner duct $(|y|<a, x<0)$ is decreased. We can see similar behavior in Figure 3.

Figure 5 depicts the graph of $|R|$ against $k$. We consider different dimensions of the coaxial region $(x<0,|y|<b) b=3$ and $b=2$, while $a=1$ and $d=4$ are kept fixed over frequency range $0<k<\pi$. Two modes can propagate in the forward direction $x>0$. The cut-off(on) frequencies do not change as the width of the inner most region is fixed. The upper bound of the reflected field coefficient decreases from 0.98 to 0.71 when the width of the coaxial region 'b' increases from 2 to 3 as shown in Figure 5.

Similarly, we present the graph of the reflected field amplitude versus the wave number $k$ in Figure 6. We have fixed the values of $a=1$ and $b=2$, while the values of ' $\mathrm{d}$ ' are 3 and 4 , 


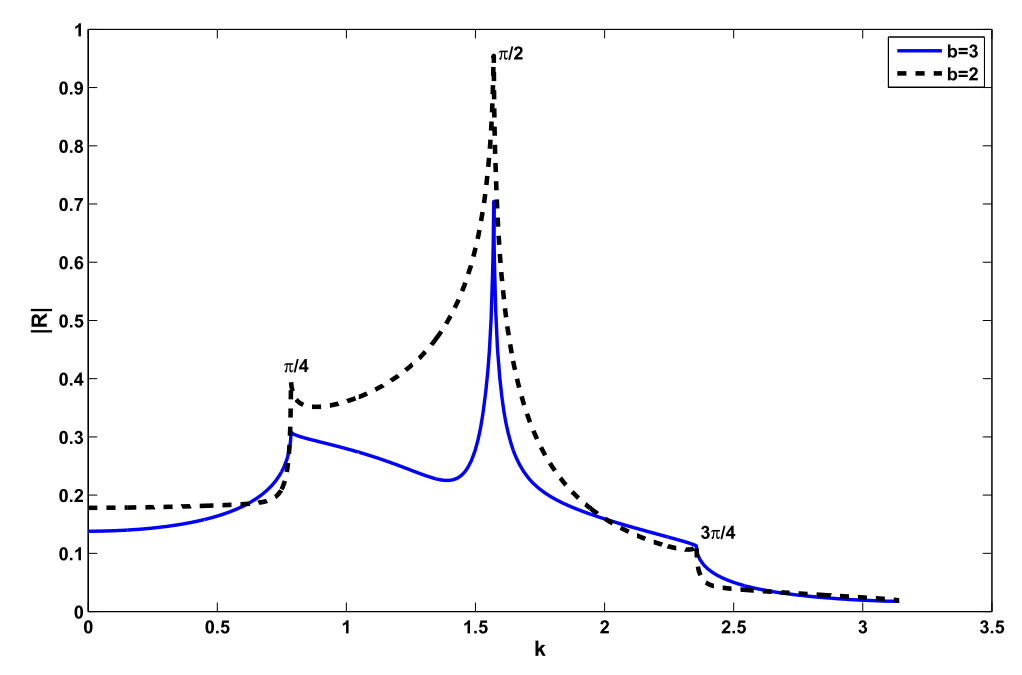

Figure 5 Reflected field amplitude $|R|$ versus the wave number $k$ for $a=1$ and $d=4$.

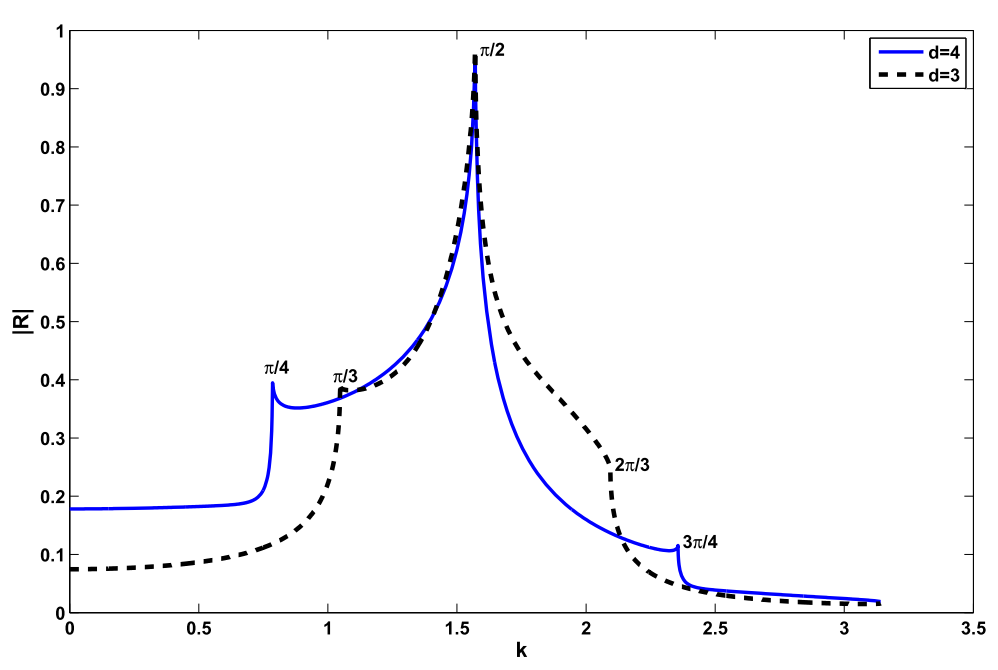

Figure 6 Reflected field amplitude $|R|$ versus the wave number $k$ for $a=1$ and $b=2$.

and they are represented by dashed line and solid line, respectively. The frequency range is $0<k<\pi$. We observe similar behavior of the reflection coefficient. We observe two modes propagate in the forward direction.

\section{Comparison}

Here, we present the comparison of the reflection coefficient results for the current problem and the previous related work on a hard pentafurcated duct [21]. We consider different dimensions for duct spacing for both problems and explain these results in two situations as mentioned below.

\subsection{Situation 1}

We consider the duct dimensions for both ducts as $a=1, b=2$ and $d=4$ over the frequency range $0<k<\pi$. Figure 7 is the graphical view of this situation. We observe clearly 


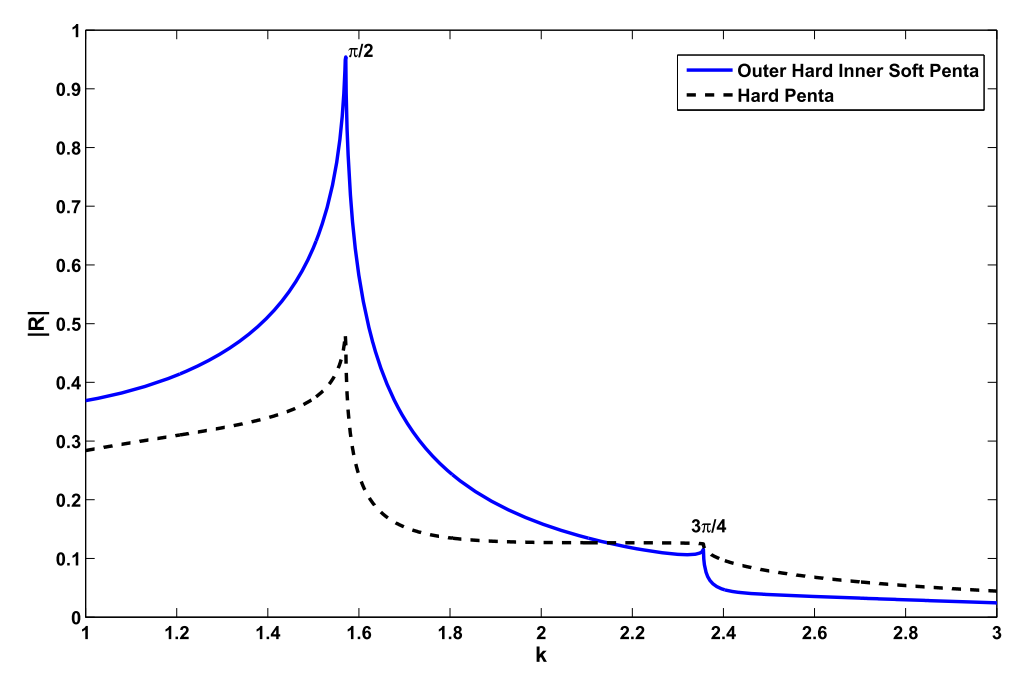

Figure 7 Reflected field amplitude $|R|$ versus the wave number $k$ for two pentafurcated problems for $a=1, b=2$ and $d=4$.

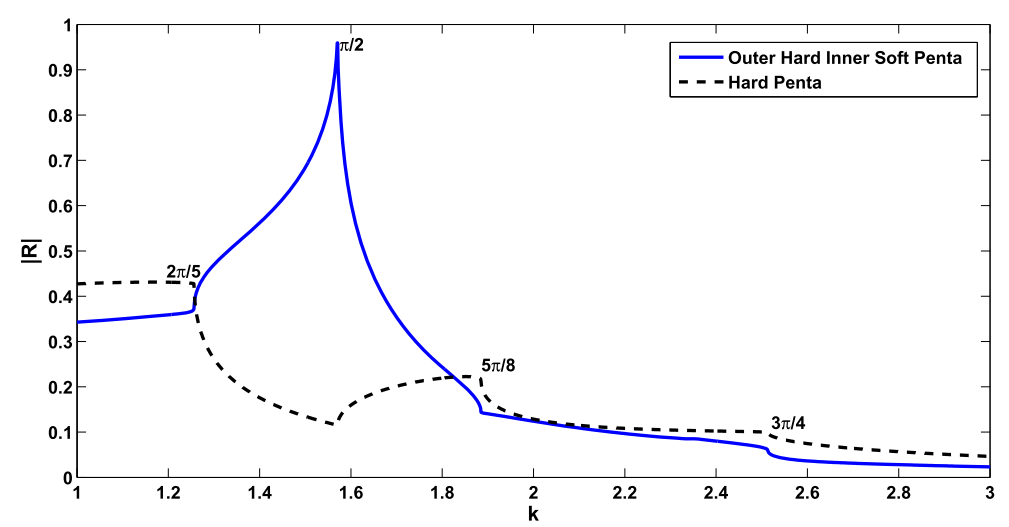

Figure 8 Reflected field amplitude $|R|$ versus the wave number $k$ for two pentafurcated problems for $a=1, b=3$ and $d=5$.

that the reflection coefficient decreases monotonically from the cut-off(on) frequency $\pi / 2$ to $3 \pi / 4$.

The behaviors of the reflected field in outer hard inner soft penta and the hard penta are similar for these dimensions. The reflection coefficient for the hard penta duct is smaller than outer hard inner soft penta for $0<k<3 \pi / 4$. We also observe that the reflection coefficient for both problems decreases to small values eventually.

\subsection{Situation 2}

Figure 8 depicts the comparison of reflection coefficient results for $a=1, b=3$ and $d=5$. The frequency range is $0<k<\pi$. The reflection coefficient decreases monotonically rapidly for the given problem from cut-off(on) frequency $\pi / 2$ to onwards $\pi$. In this case, the reflection behavior for hard penta duct [21] is quite different for $2 \pi / 5<k<5 \pi / 8$. The upper bound of the reflection coefficient for hard penta is 0.43 , while the upper bound is 1 for the given problem. 


\section{Energy conservation}

Now we use the Green's identity [26]

$$
\iint_{D}\left(\left(\nabla^{2} v+k^{2} v\right) v^{*}-\left(\nabla^{2} v^{*}+k^{2} v^{*}\right) v\right) d s=\oint_{\partial D}\left(v^{*} \frac{\partial v}{\partial x}-v \frac{\partial v^{*}}{\partial x}\right) d y=0
$$

to represent the reflected and transmitted waves energy conservation relationship with incident wave. In the given problem, $v(x, y)$ is the solution of equation (2.3) which satisfies boundary conditions (2.4)-(2.6), where $*$ indicates the conjugate and $D$ is the region of the duct $(-d<y<d,-\infty<x<\infty)$ having cuts for the semi-infinite ducts. We consider one wave that propagates in each of five regions I-V $(-d<y<d, x<0)$. Then, after the use of a little algebra, it is easy to show energy conservation relationship (7.2) from equation (7.1).

$$
\hat{\gamma}_{1}\left(1-\left|C_{1}\right|^{2}\right)=\hat{\alpha}_{1}\left|A_{1}\right|^{2}+\hat{\beta}_{1}\left|B_{1}\right|^{2}+\hat{\beta}_{1}\left|D_{1}\right|^{2}+\hat{\alpha}_{1}\left|E_{1}\right|^{2}+\sum_{j=1}^{2} \hat{\lambda}_{j}\left|F_{1}\right|^{2},
$$

where $\hat{\gamma}_{1}\left(1-\left|C_{1}\right|^{2}\right)$ represents acoustic energy which is proportional to $\left(1-|R|^{2}\right)$ that belongs to the middle region $(-a<y<a, x<0)$. This radiated energy is distributed over different regions.

\section{Final remarks}

We have investigated an outer hard inner soft pentafurcated exhaust problem by applying the straightforward mode-matching technique. This article will be helpful for engineers to design various exhaust systems for reduction of noise problems. The dominant mode is assumed to propagate in the inner region $(|y|<a, x<0)$ of the duct.

We have solved an infinite system of equations and observed that the solution becomes insensitive for $M \geq 70$ for given dimensions of the duct spacing (see Figure 2). So we can truncate at $M \approx 100$, which shows errors within line width of error plots. We have plotted the reflected field $|R|$ against the wave number $k$ for the given problem for different physical situations in Figures 3-6. We have considered different dimensions of the duct while the frequency range is $0<k<\pi$. We have observed that the reflection coefficient decreases from cut-off frequency of the backward directed propagating modes (see Figures 3 and 4). The value of $|R|$ decreases when we increase the width of the inner region $(|y|<a, x<0)$. In both Figures 5 and 6, the width of the middle region $(|y|<a, x<0)$ is kept fixed. In Figure 5, we have observed that the cut-off (on) frequency remains the same when we change the width of the coaxial region $(|y|<b, x<0)$. We have observed that the reflection increases as we decrease the duct spacing. When we change the width of the outer region $(|y|<d)$ (see Figure 6), the cut-off (on) frequency $k=\pi / 2$ does not change, but the other cut-off (on) frequencies do change. In Figures 3-6, we have observed that the value of reflected field coefficient is one at certain values of wave number $(k=\pi / 4, \pi / 2, \pi)$, which corresponds to cut-on frequencies in region III. This corresponds to maximum attenuation downstream.

In Section 6, we have compared the reflected field results against the wave number for two pentafurcated ducts with different boundary conditions (see Figures 7 and 8). We have assumed that only one wave propagates in each of the coaxial regions $(|y|<d, x<0)$. We have noticed that the value of the reflected coefficient is greater for the given problem 
than the hard pentafurcated duct problem [21] for the given frequency range (see Figures 7 and 8$)$. Thus the radiated acoustic energy which is proportional to $\left(1-|R|^{2}\right)$ for the given problem is smaller than the transmitted energy for hard pentafurcated duct. We have concluded that the inner soft surfaces provide an upper bound $(|R|=1)$ to reduce noise effects. This geometry can be solved by using the Wiener-Hopf technique, but it would require tedious, complicated calculations and computational work if compared to this technique. Future work will be extended to solving the given model in the case of fluid flow. This research work will be helpful for engineers and physicists to form the type of compressors, generators, exhaust fans and grinding mills which reduce unwanted noise in urban areas.

Competing interests

Authors have no competing interests.

Authors' contributions

All authors have equally contributed.

Acknowledgements

Authors are grateful to the referees for their painstaking review.

\section{Publisher's Note}

Springer Nature remains neutral with regard to jurisdictional claims in published maps and institutional affiliations.

Received: 12 December 2016 Accepted: 25 April 2017 Published online: 10 May 2017

\section{References}

1. Buyukaksoy, A, Tayyar, IH, Uzgoren, G: Influence of the junction of perfectly conducting and impedance parallel plate semi-infinite waveguides to the dominant mode propagation. J. Math. Anal. Appl. 143, 341-357 (2006)

2. Rawlins, AD: A bifurcated waveguide problem. J. Appl. Math. 54, 59-81 (1995)

3. Mahmood-ul-Hassan, Rawlins, AD: Two problems of waveguide carrying mean flow. J. Sound Vib. 216(4), 713-738 (1999)

4. Mahmood-ul-Hassan, Rawlins, AD: Sound radiation in a planar trifurcated lined duct. Wave Motion 29, 157-174 (1998)

5. Ayub, M, Tiwana, MH, Mann, AB, et al.: Acoustic wave propagation in a trifurcated lined waveguide. ISRN Appl. Math. 2011, Article ID 532682 (2011)

6. Ayub, M, Tiwana, MH, Mann, AB: Wiener-Hopf analysis of an acoustic plane wave in a trifurcated waveguide. Arch. Appl. Mech. 81, 701-713 (2011)

7. Ayub, M, Tiwana, MH, Mann, AB: Influence of the dominant mode propagation in a trifurcated lined duct with different impedance. Phys. Scr. 81(3), Article ID 035402 (2010)

8. Demir, A, Cinar, OY: Propagation of sound in an infinite two-part duct carrying mean flow inserted axially into a large infinite duct with wall impedance discontinuity. J. Appl. Math. Mech. 89(6), 454-465 (2009)

9. Andronov, IV, Belinskii, BP: On acoustic boundary-contact problems for a vertically stratified medium bounded from above by a plate with concentrated inhomogeneities. J. Appl. Math. Mech. 54, 366-371 (1990)

10. Lawrie, JB, Abrahams, ID: An orthogonality condition for a class of problems with high order boundary conditions, application in sound/structure interaction. Q. J. Mech. Appl. Math. 52, 161-181 (1999)

11. Lawrie, JB, Kirby, R: Mode-matching without root-finding: application to a dissipative silencer. J. Acoust. Soc. Am. 119 2050-2061 (2006)

12. Lawrie, JB: On eigenfunction expansions associated with wave propagation along ducts with wave bearing boundaries. IMA J. Appl. Math. 72, 376-394 (2007)

13. Lawrie, JB: Analytic mode-matching for acoustic scattering in three dimensional waveguides with flexible walls, application to a triangular duct. Wave Motion 50(3), 542-557 (2013)

14. Mahmood-ul-Hassan, Meylan, MH, Peter, MA: Water-wave scattering by submerged elastic plates. Q. J. Mech. Appl. Math. 62(3), 321-344 (2009)

15. Nawaz, R, Lawrie, JB: Scattering of a fluid-structure coupled wave at a flanged junction between two flexible waveguides. J. Acoust. Soc. Am. 134(3), 1939-1949 (2013)

16. Decrossas, E, Sabbagh, MAE, Hanna, VF, El-Ghazaly, SM: Mode matching technique-based modeling of coaxial and circular waveguide discontinuities for material characterization purposes. Int. J. Microw. Wirel. Technolog. 3(6), 679-690 (2011)

17. Ranajit, D, Chakarbarty, S, Ranjeev, J, et al.: Synthesis and analysis of multi-mode profile horn using mode-matching technique and evolutionary algorithm. IET Microw. Antennas Propag. 10(3), 1-7 (2016)

18. Meylan, MH, Bennets, LG, Peter, MA: Water-wave scattering and energy dissipation by a floating porous elastic plate in three dimensions. Wave Motion 70, 240-250 (2017). doi:10.1016/j.wavemoti.2016.06.014

19. Sanchis, P, Marti, J, Balasco, J, Martinez, A, et al.: Mode matching technique for highly efficient coupling between dielectric waveguides and planar photonic crystal circuits. Opt. Express 10, 1391-1397 (2003)

20. Mahmood-ul-Hassan: Wave scattering by soft-hard three spaced waveguide. Appl. Math. Model. 38, 4528-4537 (2014) 
21. Mahmood-ul-Hassan, Meylan, MH, Amna, B, et al.: Mode matching analysis for wave scattering in triple and pentafurcated spaced ducts. Math. Methods Appl. Sci. 39(11), 2783-3158 (2016)

22. Mahmood-ul-Hassan, Mahvish, N, Nawaz, R: Reflected field analysis of soft-hard pentafurcated waveguide. Adv. Mech. Eng. 9(2), 1-11 (2017). doi:10.1177/1687814017692697

23. Afzal, M, Nawaz, R, Ayub, M, Wahab, A: Acoustic scattering in flexible waveguide involving step discontinuity. PLoS ONE 9(8), e103807 (2014)

24. Mei, CC (ed.): The Applied Dynamics of Ocean Surface Waves. Wiley, New York (1983)

25. Linton, CM, Mclver, P (eds.): Handbook of Mathematical Techniques for Wave/Structure Interactions. Chapman and Hall/CRC, New York (2001)

26. Riley, KF, Hobson, MP, Bence, SJ: Mathematical Methods for Physics and Engineering, 3rd edn. Cambridge University Press, Cambridge (2006)

Submit your manuscript to a SpringerOpen ${ }^{\odot}$ journal and benefit from:

- Convenient online submission

Rigorous peer review

- Immediate publication on acceptance

- Open access: articles freely available online

- High visibility within the field

- Retaining the copyright to your article 